\title{
Perspectives
}

\section{Molecular Genetics of the Cutaneous Basement Membrane Zone Perspectives on Epidermolysis Bullosa and Other Blistering Skin Diseases}

\author{
Jouni Uitto and Angela M. Christiano \\ Departments of Dermatology, and Biochemistry and Molecular Biology, Jefferson Medical College, Section of Molecular Dermatology, \\ Jefferson Institute of Molecular Medicine, Thomas Jefferson University, Philadelphia, Pennsylvania 19107
}

\section{Introduction}

Considerable progress has recently been made in understanding the molecular basis of the cutaneous basement membrane zone $(\mathrm{BMZ})^{1}$ with respect to several heritable and acquired diseases of the skin. One of these diseases is epidermolysis bullosa (EB), a heterogeneous group of conditions characterized by the formation of blisters and erosions of the skin and mucous membranes in response to trauma $(1,2)$. The clinical and genetic heterogeneity of EB is reflected by the large number of subtypes which have been delineated to reflect salient features of the clinical phenotype and the mode of inheritance (1). In the simplest form of classification, EB can be divided into three major categories on the basis of the level of tissue separation (Fig. 1), as demonstrated by diagnostic electron microscopy. (a) In the simplex, nonscarring forms of EB, the blister formation occurs intraepidermally at the level of the basal keratinocytes; $(b)$ in the junctional forms of EB, the tissue separation occurs within the dermal-epidermal basement membrane at the level of the lamina lucida; $(c)$ in the dystrophic, scarring forms of EB, the tissue separation occurs below the basement membrane within the upper dermis at the level of the anchoring fibrils ( Table I). Both autosomal dominant and autosomal recessive patterns of inheritance can be recognized $(1,2)$.

In addition to the heritable subtypes, epidermolysis bullosa acquisita (EBA), an acquired autoimmune disease in which the patient's serum contains autoantibodies directed against type VII collagen, results in a clinical presentation similar to that of the inherited dystrophic forms of $E B(3)$. In addition to EBA, several different blistering skin diseases have an autoimmune pathogenesis, and circulating autoantibodies recognizing cutaneous epitopes have been demonstrated in patients'

Address correspondence to Jouni Uitto, M.D., Ph.D., Department of Dermatology, Jefferson Medical College, 233 South 10th Street, BLSB 450, Philadelphia, PA 19107.

Received for publication 9 June 1992.

1. Abbreviations used in this paper: $\mathrm{BMZ}$, basement membrane zone; BP, bullous pemphigoid; BPAG1 and 2, BP antigen 1 and 2; COL7A1, type VII collagen gene; DDEB, dominantly inherited forms of DEB; DEB, dystrophic forms of epidermolysis bullosa; EB, epidermolysis bullosa; EBA, EB acquisita; EBS, simplex forms of EB; PV, pemphigus vulgaris; RDEB, recessively inherited forms of DEB; RFLP, restriction fragment length polymorphism.

J. Clin. Invest.

(C) The American Society for Clinical Investigation, Inc.

$0021-9738 / 92 / 09 / 0687 / 06 \$ 2.00$

Volume 90, September 1992, 687-692 sera. These acquired conditions include bullous pemphigoid (BP) and pemphigus vulgaris (PV), among others (see Table I).

The cutaneous $\mathrm{BMZ}$ consists of a large number of distinct macromolecules that form an intricate network at the dermalepidermal interface through discrete molecular interactions (Fig. 1). Some of the components of the cutaneous basement membrane zone, such as type IV collagen, laminin and nidogen, are expressed ubiquitously in basement membranes throughout the body (4). However, some structural macromolecules are present exclusively in the $\mathrm{BMZ}$ of stratifying squamous epithelia, i.e., the skin, mucous membranes, and the cornea of the eye (2). The presence of a large number of structural components in the cutaneous basement membrane zone suggests that several genes could potentially serve as candidate genes in the various forms of EB and other cutaneous blistering diseases ( Table I).

Over the past two decades, much of our understanding of the general structural features of basement membrane components has been derived from neoplastic animal tissues, such as the murine EHS tumor, which produce large quantities of $\mathrm{BMZ}$ macromolecules ( 3 ). In contrast, the low abundance and relative insolubility of the cutaneous $\mathrm{BMZ}$ components in normal human tissues have largely precluded extensive characterization of these molecules at the protein level. More recently, however, molecular cloning of many of the human genes corresponding to the BMZ components has allowed precise elucidation of the primary sequences of structural macromolecules of the cutaneous BMZ.

This overview will highlight recent advances in the molecular genetics of the cutaneous BMZ in relation to pathomechanisms of blistering skin diseases.

\section{Molecular biology of the hemidesmosomal proteins}

One of the characteristic features of the BMZ of stratifying squamous epithelia is the presence of hemidesmosomes, attachment structures which reside at the basal keratinocytelamina lucida interface (Fig. 1). Immunoelectron microscopy has topographically localized at least three protein components to the hemidesmosomes. They include bullous pemphigoid antigen 1 (BPAG1), a 230-kD noncollagenous protein which is the major autoantigen in BP, a blistering autoimmune disease (5). Another hemidesmosomal protein is bullous pemphigoid antigen 2 (BPAG2), a 180-kD collagenous protein which serves as an autoantigen in some patients with $\mathrm{BP}$, and also in herpes gestationis, a blistering disease associated with pregnancy (6). Several lines of evidence indicate that the two bullous pemphigoid antigens are clearly distinct gene products. Specifically, cloning of the corresponding cDNAs has indicated 


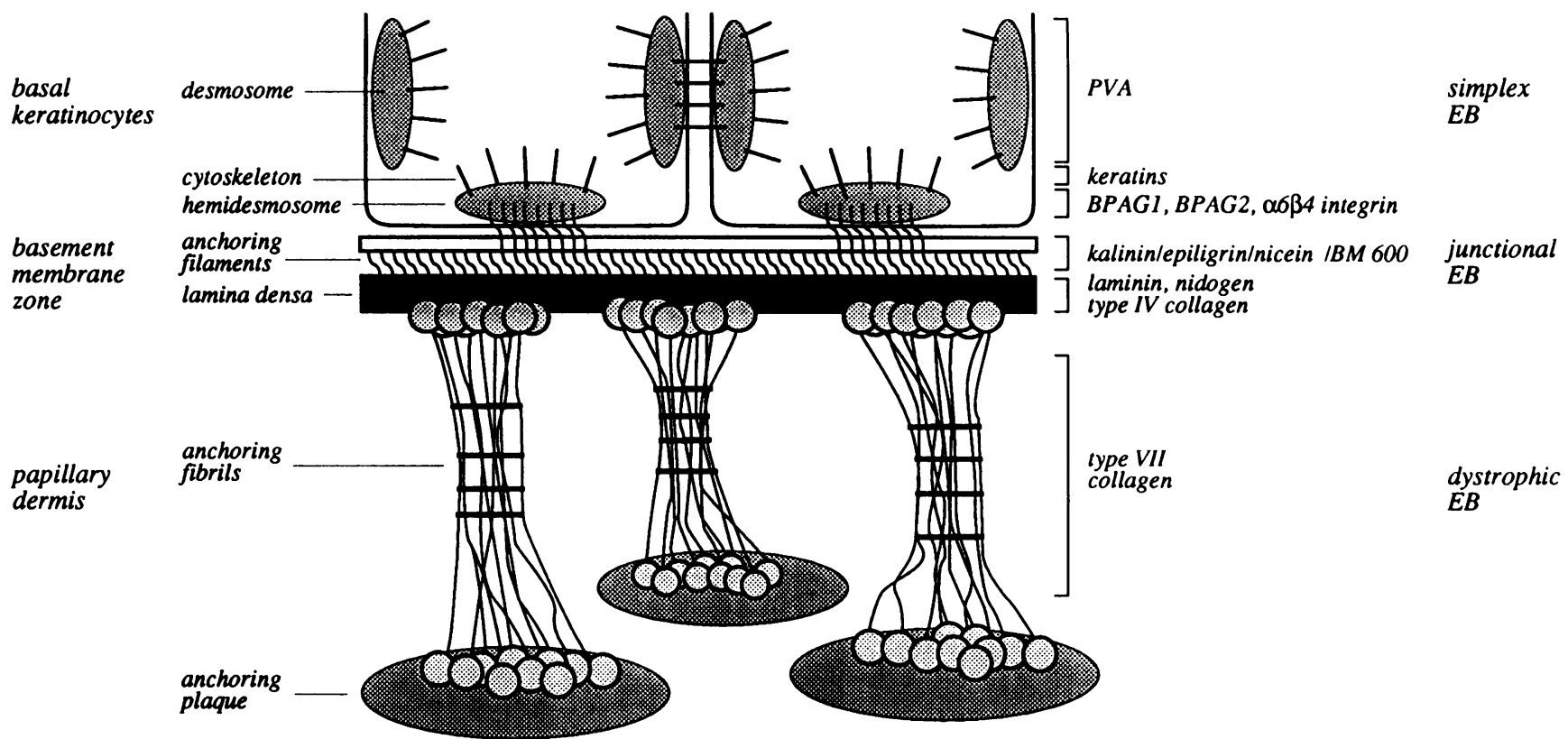

Figure 1. Schematic representation of the organization of the cutaneous basement membrane zone. As shown on the left, the figure depicts basal keratinocytes overlying the papillary dermis, with the basement membrane zone separating the two compartments. The presence of ultrastructurally recognizable morphologic structures are indicated on the left, and the individual proteins comprising these components are indicated on the right. Also note the level of tissue separation in the simplex, junctional, and dystrophic forms of EB.

that there is no sequence homology between the primary sequences of these proteins, as deduced from the nucleotide sequences (7-9). Furthermore, the genes for BPAG1 and BPAG2 have been mapped to two different chromosomes, viz. to the short arm of chromosome 6 and the long arm of chromosome 10 , at loci $6 \mathrm{p} 11-12$ and 10q24, respectively $(10,11)$.

The third protein associated with the hemidesmosomal complexes is $\alpha 6 \beta 4$ integrin, an attachment protein which is expressed by epithelial cells (12). The $\alpha 6 \beta 4$ integrin has been shown to be polarized to the underside of epidermal keratinocytes, on the surface apposed to the basement membrane, where it has been postulated to mediate the attachment of hemi- desmosomes to the underlying BMZ. Since previous studies have suggested that BPAG1 is largely intracellular and BPAG2 is a transmembrane protein, while $\alpha 6 \beta 4$ integrin may reside largely in the extracellular space, these observations suggest that the hemidesmosomal proteins form a continuous link which secures the attachment of the basal keratinocytes to the underlying basement membrane (2).

The pathoetiologic role of circulating autoantibodies in EBA and BP, recognizing type VII collagen and BP antigen epitopes, respectively, is still unclear. In contrast, the circulating $\mathrm{IgG}$ antibodies in $\mathrm{PV}$ are clearly pathogenetic. Amagai et al. (13) have convincingly demonstrated that IgG antibodies rec-

Table I. Molecular Basis of Blistering Skin Diseases

\begin{tabular}{|c|c|c|c|c|}
\hline Disease & $\begin{array}{l}\text { Level of tissue* } \\
\text { separation }\end{array}$ & $\begin{array}{c}\text { Candidate }{ }^{\ddagger} \\
\text { genes/proteins }\end{array}$ & $\begin{array}{l}\text { Chromosomal locus } \\
\text { of the gene }\end{array}$ & $\begin{array}{c}\text { See } \\
\text { reference }\end{array}$ \\
\hline \multicolumn{5}{|l|}{ Heritable diseases } \\
\hline EB simplex & Basal keratinocytes & Keratins $5^{*}$ and $14^{*}$ & $12 \mathrm{q}, 17 \mathrm{q}$ & 25 \\
\hline EB junctional & Lamina lucida & Anchoring filament proteins (see text) & $\mathrm{ND}^{\S}$ & \\
\hline EB dystrophic & Sub-lamina densa & Type VII collagen* & $3 \mathrm{p} 21$ & 20 \\
\hline Epidermolytic hyperkeratosis & Intraepidermal, suprabasilar & Keratins $1^{*}$ and 10 & $12 \mathrm{q}, 17 \mathrm{q}$ & 42 \\
\hline \multicolumn{5}{|l|}{ Acquired diseases } \\
\hline EB acquisita & Sub-lamina densa & Type VII collagen & $3 \mathrm{p} 21$ & 20 \\
\hline Bullous pemphigoid & Lamina lucida & BPAG1 and/or BPAG2 & $6 \mathrm{p} 11,10 \mathrm{q} 24$ & 10,11 \\
\hline Herpes gestationis & Lamina lucida & BPAG2 & $10 q 24$ & 11 \\
\hline Pemphigus vulgaris & Intraepidermal, subrabasilar & PVA $^{\S}$ (cadherin) & 18 & 43 \\
\hline Pemphigus foliaceus & Intraepidermal, superficial & Desmoglein I (cadherin) & 18 & 43 \\
\hline
\end{tabular}

* For structural details of the BMZ, see Fig. 1.

¥ Specific mutations or genetic linkage have been demonstrated in heritable diseases in the genes marked by an asterisk. The proteins indicated under acquired diseases serve as autoantigens.

$\S$ PVA, PV antigen; ND, not determined as yet. 
ognizing an antigenic region in the PV antigen, a cell surface cadherin, are capable of producing a suprabasilar acantholysis when injected to the skin of neonatal mice.

\section{Anchoring filament proteins}

In the junctional forms of EB, the tissue cleavage occurs within the lamina lucida, in the region of skin-specific structures known as anchoring filaments $(1,2)$. Considerable progress has recently been made in understanding the structure of anchoring filament proteins. Initially, a monoclonal antibody (GB3) was shown to recognize a large glycoprotein, BM600, which appears to be exclusively expressed in the cutaneous basement membrane zone and particularly within the lamina lucida (14). The BM600 protein, which has recently been renamed nicein, consists of three disulfide linked polypeptide subunits that constitute an $\sim 600-\mathrm{kD}$ protein. Recently, characterization of two additional basement membrane zone proteins, kalinin and epiligrin, revealed structural and biological features remarkably similar to nicein $(15,16)$. It is conceivable, therefore, that these three proteins are identical or closely related.

Epitopes for nicein/kalinin/epiligrin have been localized to the anchoring filaments within the lamina lucida by immunoelectron microscopy (15). Since immunostaining of skin from patients with the junctional forms of EB with the GB3 antibody, as well as with another monoclonal antibody 19DEJ1 (2), shows little immunoreactivity, while the staining pattern is normal in other forms of EB, it is conceivable that the corresponding gene(s) are candidates in the junctional forms of EB. Although no direct evidence for a mutation in nicein/ kalinin/epiligrin has been demonstrated as yet, this hypothesis can be tested by direct analysis of the primary sequences of these gene(s) in patients with the junctional forms of EB as soon as the normal sequences are available.

\section{Molecular genetics of type VII collagen}

The anchoring fibrils are morphologically distinct attachment structures that extend from the lower portion of the basement membrane to the papillary dermis (Fig. 1). Several independent lines of evidence suggest that the anchoring fibrils play a critical role in stabilizing the association of the lower part of the basement membrane to the underlying dermis (17).

Type VII collagen is present in human tissues in a limited pattern of distribution, and specifically, it is found almost exclusively in the basement membrane zone below the stratifying squamous epithelia of the skin, mucous membranes, and the cornea of the eye (18). This collagen is the major component of anchoring fibrils, as demonstrated by immunolocalization studies. Type VII collagen consists of three identical $\alpha$-chains, $[\alpha 1 \text { (VII) }]_{3}$, and each $\alpha$-chain is synthesized as a precursor polypeptide, pro $\alpha 1$ (VII) chain (19). Each pro $\alpha$-chain, $\sim 300 \mathrm{kD}$ in size, consists of a central collagenous domain that accounts for about half of the mass of the molecule, a larger amino-terminal noncollagenous domain, NC-1, and a smaller carboxyterminal noncollagenous domain, NC-2 (Fig. 2). In the extracellular space, the individual type VII collagen molecules form an antiparallel dimer through overlapping carboxy-terminal sequences (Fig. 2). Multiple dimer molecules then assemble laterally to form anchoring fibrils that contain large globular NC-1 domains at both ends of the structure (19). It has been suggested that the NC-1 domains interact at one end with components of the dermal-epidermal basement membrane, and at
1
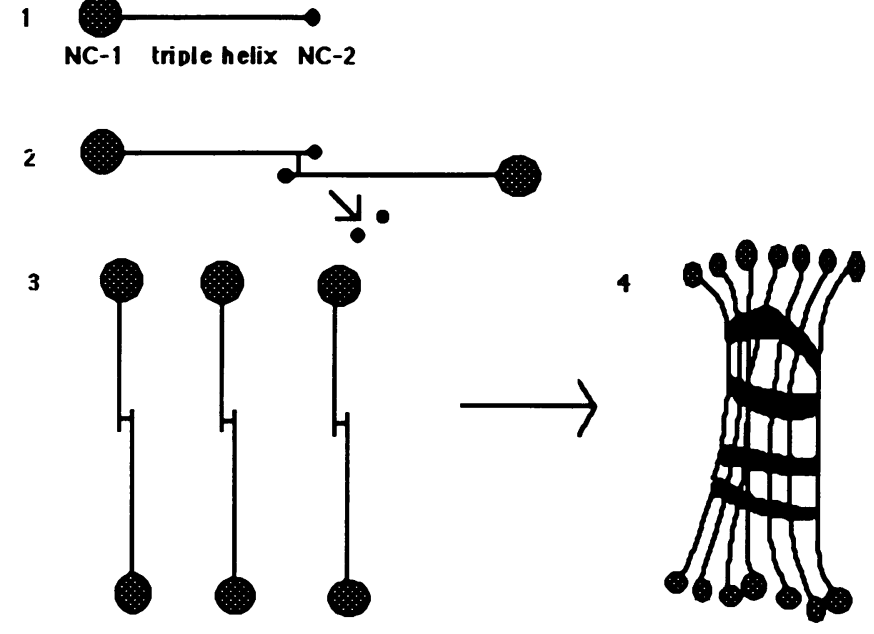

Figure 2. Schematic representation of type VII collagen and its assembly to anchoring fibrils. 1. Type VII collagen consists of a central triple-helical domain flanked by an amino-terminal noncollagenous domain, NC-1, and a carboxy-terminal noncollagenous segment, NC-2. 2. In the extracellular space, two procollagen molecules align to form antiparallel dimers which are stabilized by the formation of disulfide bonds. 3 . The NC-2 domain is proteolytically cleaved to yield stable type VII collagen dimers. 4. Several of these dimer molecules laterally assemble into anchoring fibrils which can be recognized by their characteristic banding pattern.

the other end with islands of basement membrane-like structures, known as anchoring plaques, within the upper dermis (Fig. 1). Also, some of the anchoring fibrils form U-shaped structures with both NC-1 domains attached to the lamina densa, so that the anchoring fibrils entrap other components of the dermal connective tissue, such as the broad, banded collagen fibrils consisting primarily of type I and III collagens. These arrangements securely bind the cutaneous basement membrane to the underlying dermis, providing stability to the lower portion of the basement membrane zone (2).

Elucidation of the primary sequence of the pro $\alpha 1$ (VII)chain has recently been advanced by cloning of human type VII collagen cDNAs $(20,21)$. These studies have revealed several intriguing features within the type VII collagen molecule. For example, the nucleotide sequences unequivocally predict that the NC-1 domain is amino-terminal with respect to the central collagenous domain, while NC-2 resides at the carboxyterminal end of the molecule. This domain assignment contrasts the previous model which was based on preliminary biochemical information of type VII collagen (19). Furthermore, elucidation of the primary sequences within the collagenous domain has revealed the presence of characteristic Gly-X-Y repeats, which are frequently interrupted by noncollagenous imperfections (17). It is conceivable that such interruptions provide flexibility to the molecule, as predicted from studies using biophysical parameters and rotary shadowing electron microscopy (22). Such flexibility may be necessary for the anchoring fibrils to function as the attachment structures at the lamina densa/papillary dermis interface.

Elucidation of the primary sequences of the pro $\alpha 1$ (VII) collagen chain has revealed features that may be of biological importance. These include the presence of several RGD tripeptide sequences potential cell binding sites. Furthermore, the 
NC-1 domain contains nine segments with homology to fibronectin type III motifs, as well as subdomains with homology to the A domain of von Willebrand factor. These sequences predict that adhesive properties of the NC-1 domain of type VII collagen may play a role in cell-matrix interactions.

Localization of the type VII collagen gene (COL7A1) to a chromosomal locus on the short arm of human chromosome 3 and identification of two informative polymorphisms within the gene have facilitated genetic linkage analyses in families with the dystrophic forms of $\mathrm{EB}(20,21,23)$.

\section{Genetic linkage analyses and keratin mutations in epidermolysis bullosa simplex}

Remarkable progress has recently been made in understanding the molecular basis of the simplex forms of EB (EBS). These nonscarring forms of EB are inherited in an autosomal dominant manner in the majority of cases (1). Initial ultrastructural observations indicated that clumping of intracellular tonofilaments occurred in association with blister formation in some forms of EBS. Subsequent linkage analyses mapped several EBS loci to either chromosome 12 or chromosome 17, in the vicinity of gene clusters known to encode keratins expressed in the epidermis (24-26). Specifically, keratin 5 on chromosome 12 and keratin 14 on chromosome 17 are exclusively expressed in the basal keratinocytes, where they assemble into heterodimeric filaments necessary for the integrity of the basal cell cytoskeleton (27). Further support to the hypothesis that abnormalities in the keratin genes may underlie EBS was generated by creation of a series of transgenic mice into which truncated constructs of the keratin 14 gene had been integrated (28). The transgenic animals developed a clinical disease characterized by intraepidermal blistering at birth, and ultrastructural evidence suggested clumping of tonofilaments. Subsequently, specific mutations have been demonstrated in patients with EBS. In particular, in two patients from different families with EBS herpetiformis (the Dowling-Meara variant), there was a point mutation in codon 125 , which normally encodes an arginine residue (25). In one case, the mutation changed the arginine to cysteine, while in the other, the arginine was changed to histidine. Another family with the Köbner-type of EBS, with generalized distribution of lesions, has been shown to have a point mutation in keratin 14 resulting in substitution of a leucine residue by a proline at position 384 (25). In another keratinization disorder, epidermolytic hyperkeratosis, characterized by intraepidermal blistering, suprabasilar keratins, 1 and 10 have been implicated in the disease process (Table I). Although the precise molecular consequences of these keratin mutations are as yet unclear, these mutations apparently result in poor intermediate filament formation, causing fragility of keratinocytes and manifesting as tissue separation within the epidermis.

\section{Evidence for type VII collagen as the candidate gene in the dystrophic forms of $E B$}

Previous studies have clearly demonstrated abnormalities in the anchoring fibrils in the dystrophic forms of EB (DEB) (2). In particular, electron microscopy of the skin in patients with the dominantly inherited forms of DEB (DDEB) has demonstrated that the anchoring fibrils are reduced in number and/or are morphologically altered $(17,29)$. In the more severe, recessively inherited forms of DEB (RDEB), the anchoring fibrils can be entirely absent or only a few severely abnormal fibril structures can be detected (30). Since type VII collagen is the major component of the anchoring fibrils, it has been postulated that COL7A1 is the candidate gene for mutations in families with the dystrophic forms of EB.

This hypothesis has recently been tested by genetic linkage analyses $(23,29)$. To provide informative markers for these studies, we initially identified a PvulI restriction fragment length polymorphism (RFLP) which was shown by direct sequencing of the genomic region to reflect an $A$ to $G$ transition in the third position of a proline codon $(23,31)$. This PvulI RFLP was initially applied to a large Finnish kindred with 20 affected and 23 unaffected living individuals in four generations, to examine the segregation of COL7A1 alleles with the DDEB phenotype (23). Cosegregation of the RFLP marker and the clinical phenotype was observed in this family, demonstrating strong genetic linkage with the maximum Lod score ( $\hat{Z})$ of 6.5 at a recombination fraction $\theta=0(23,29)$. The strong genetic linkage $(\hat{Z}>3)$ and the absence of recombination between the COL7A1 and DDEB loci, as well as demonstration of anchoring fibril defects in the affected individuals, strongly suggested that the type VII collagen gene harbors the mutations causing the blistering disease in this family.

Subsequent to the initial demonstration of linkage between COL7A1 and DDEB, several additional kindreds of diverse ethnic background, with clinical and genetic features diagnostic of DDEB, have been examined (32). Strong genetic linkage in families of Finnish, Dutch, Japanese, and U.S. Caucasian extraction has been observed with COL7A1 and several informative flanking markers $(29,32)$. Thus, the linkage data indicate a single locus on chromosome $3 p$ as the site of the dominant dystrophic EB mutation(s), with no evidence for genetic heterogeneity thus far.

Previous studies have also indicated that the RDEB are characterized by morphologic abnormalities or by the absence of anchoring fibrils (30). Thus, it is conceivable that type VII collagen is also a candidate gene for mutations in families with RDEB. This hypothesis has recently been tested in a study by Hovnanian et al. (33), which examined 24 kindreds with at least one individual presenting with a mutilating form of RDEB. In five of these pedigrees, there were two or more affected individuals, and consanguinity was documented in three families. The parents of the affected individuals were clinically normal in all cases. Thus, the appearance of the clinical phenotype in these individuals is consistent with a recessively inherited mutation. Genetic linkage analyses using the PvuII RFLP within COL7A1 as a marker indicated cosegregation of a particular type VII collagen allele and the clinical phenotype in several of these families, with a combined maximum Lod score $(\hat{Z})$ of 3.97 at a recombination fraction $(\theta)$ zero (33). These data strongly support the possibility that type VII collagen is the primary candidate gene for mutations in both the dominantly and recessively inherited forms of DEB. Previous studies have suggested that proteolytic enzymes, such as collagenase, may play a role particularly in RDEB (34). It should be noted, however, that recent studies have excluded the collagenase locus on chromosome 11 as the site of the primary mutation in some families with RDEB (35).

Although the precise mutations in type VII collagen in the dystrophic forms of EB are currently under intense investigation, we speculate that mutations in COL7A1, by analogy with other collagen genes, including those for types I, II, III, and IV, will consist of a spectrum of different kinds of mutations (36). 
For example, the mutations could lead to altered expression of the gene, which would explain the low abundance of type VII collagen in dominantly inherited DEB, or might explain the complete absence of type VII collagen and anchoring fibrils in recessively inherited forms. Structural mutations in the type VII collagen gene could also introduce changes in the protein which would delay secretion of the molecules into the extracellular space, or interfere with the assembly of type VII collagen into the anchoring fibrils (30). By analogy with other collagen genes, mutations substituting critical glycine residues with other amino acids within the collagenous domain could lead to reduced stability of the collagenous triple helix, thus conferring instability to the entire type VII collagen molecule and possibly to the anchoring fibrils.

\section{Applications of gene therapy to $E B$}

The potential use of gene transfer approaches appears to be particularly well suited to cutaneous diseases characterized by abnormalities in the genes that are expressed primarily by keratinocytes. In particular, the technologies to propagate epidermal cells in culture and to use such cells as autologous grafts are well established, and are in clinical use for severe cutaneous burns and leg ulcers (37). Furthermore, such grafting procedures have been applied with promising results to selected patients with the junctional forms of EB (38). Thus, one of the future goals of research towards improved management and potential cure of various forms of EB relates to strategies that could replace the mutated gene by targeted transfection with the wild type gene in transplantable keratinocytes (39). This strategy appears to be particularly applicable to patients who carry a null allele, which is not expressed, leading to the complete absence of type VII collagen production and absence of anchoring fibrils in certain cases with the RDEB (30). In the case of dominant-negative mutations, in which the mutated gene product interferes with the function of the normal protein, the strategy of using antisense oligomers directed against expression of the mutated allele could potentially be useful. The latter approach could be combined with pharmacologic upregulation of the expression of the normal allele in the heterozygous individuals. In this regard, it is of interest to note that transforming growth factor- $\beta$ is a powerful upregulator of type VII collagen gene expression in cultures of human epidermal keratinocytes (40). Thus, cotransfection of transplantable keratinocytes with the transforming growth factor- $\beta$ gene together with the normal type VII collagen gene could lead to enhanced synthesis of type VII collagen in situ. Intriguingly, a recent study has demonstrated that direct trans-epidermal introduction of human DNA into mouse skin using a biolistic microprojectile accelerator leads to expression of the gene, as detected by development of antibodies against the human protein (41). Similar approaches could be applicable to different forms of EB once the candidate genes and the precise underlying mutations have been elucidated in detail. It is clear that approaches of molecular and cell biology in combination with molecular pharmacology will facilitate the development of strategies to counteract these often fatal and frequently devastating diseases of the skin.

\section{Acknowledgments}

The authors thank Debra Pawlicki for expert secretarial help and Dan Kuetemeyer for the assistance in illustrations. The authors acknowl- edge the participation of the following individuals in the original studies performed in the Jefferson Dermatology research laboratories: Drs. Mon-Li Chu, Linda Chung-Honet, Renato Iozzo, Robert Knowlton, Kehua Li, M. Gabriela Parente, Jaana Ryynänen, Markku Ryynänen, and Stephan Sollberg. The authors appreciate the collaboration of Drs. David Woodley, Kimberly Wynn, Eugene Bauer, and David Olsen (Stanford University) and Drs. Alain Hovnanian and Michel Goossens (Institut National de la Santé et de la Recherche Medicale, Creteil, France).

This work was supported in part by the U. S. Public Health Service, National Institutes of Health grants PO1-AR38923 and T32-AR07561. Dr. Christiano is the recipient of a Dermatology Foundation Research Fellowship.

\section{References}

1. Fine, J. D., E. A. Bauer, R. A. Briggaman, D. M. Carter, R. A. J. Eady, N. B. Easterly, K. A. Holbrook, S. Hurwitz, L. Johnson, A. Lin, et al. 1991. Revised clinical and laboratory criteria for subtypes of inherited epidermolysis bullosa. A consensus report by the subcommittee on diagnosis and classification of the $\mathrm{Na}$ tional Epidermolysis Bullosa Registry. J. Am. Acad. Dermatol. 24:119-135.

2. Uitto, J., E. A. Bauer, and A. N. Moshell. 1992. Symposium on epidermolysis bullosa: molecular biology and pathology of the cutaneous basement membrane zone. J. Invest. Dermatol. 98:391-395.

3. Woodley, D. T., R. E. Burgeson, G. P. Lunstrum, L. Bruckner-Tuderman, M. Reese, and R. A. Briggaman. 1988. Epidermolysis bullosa acquisita antigen is the globular carboxyl terminus of type VII collagen. J. Clin. Invest. 81:683-687.

4. Timpl, R., and M. Dziadek. 1986. Structure, development and molecular pathology of basement membranes. Int. Rev. Exp. Pathol. 29:1-112.

5. Stanley, J. R. 1991. Pemphigus and pemphigoid as paradigms of organ-specific, autoantibody-mediated diseases. J. Clin. Invest. 83:1443-1448.

6. Diaz, L. A., H. Ratrie III, W. S. Saunders, S. Futamura, H. L. Squiquera, G. J. Anhalt, and G. J. Giudice. 1990. Isolation of a human epidermal cDNA corresponding to the $180-\mathrm{kD}$ autoantigen recognized by bullous pemphigoid and herpes gestationis sera. J. Clin. Invest. 86:1088-1094.

7. Stanley, J. R., T. Tanaka, S. Mueller, V. Klaus-Kovtun, and D. Roop. 1988. Isolation of complementary DNA for bullous pemphigoid antigen by use of patients' autoantibodies. J. Clin. Invest. 82:1864-1870.

8. Sawamura, D., K. Li, M.-L. Chu, and J. Uitto. 1991. Human bullous pemphigoid antigen (BPAG1): amino acid sequence deduced from cloned cDNAs predicts biologically important peptide segments and protein domains. $J$. Biol. Chem. 266:17784-17790.

9. Giudice, G., H. L. Squiquera, P. M. Elias, and L. A. Diaz. 1991. Identification of two collagen domains within the bullous pemphigoid autoantigen, BP180. J. Clin. Invest. 87:734-738.

10. Sawamura, D., K. Nomura, Y. Sugita, M.-G. Mattei, M.-L. Chu, R. Knowlton, and J. Uitto. 1990. Bullous pemphigoid antigen: cDNA cloning and mapping of the gene to the short arm of human chromosome 6. Genomics. 8:722-726.

11. Li, K., D. Sawamura, G. J. Giudice, L. A. Diaz, M.-G. Mattei, M.-L. Chu, and J. Uitto. 1991. Genomic organization of collagenous domains and chromosomal assignment of human 180-kD bullous pemphigoid antigen (BPAG2), a novel collagen of stratified squamous epithelium. J. Biol. Chem. 266:2406424069.

12. Stepp, M. A., S. Spurr-Michaud, A. Tisdale, J. Elwell, and I. K. Gipson. 1990. $\alpha 6 \beta 4$ integrin heterodimer is a component of hemidesmosomes. Proc. Natl. Acad. Sci. USA. 87:8970-8974.

13. Amagai, M., S. Karpati, R. Prussick, V. Klaus-Kovtun, and J. R. Stanley. 1992. Autoantibodies against the amino-terminal cadherin-like binding domain of pemphigus vulgaris antigen are pathogenic. J. Clin. Invest. 90:919-926.

14. Verrando, P., A. Pisani, and J.-P. Ortonne. 1988. The new basement membrane antigen recognized by the monoclonal antibody GB3 is a large size weight glycoprotein: modulation of its expression by retinoic acid. Biochim. Biophys. Acta. 942:45-56.

15. Rouselle, P., G. P. Lunstrum, D. R. Keene, and R. E. Burgeson. 1991. Kalinin: an epithelium-specific basement membrane adhesion molecule that is a component of anchoring filaments. J. Cell Biol. 114:567-576.

16. Carter, W. G., M. C. Ryan, and P. J. Gahr. 1991. Epiligrin, a new cell adhesion ligand for integrin $\alpha 3 \beta 1$ in epithelial basement membranes. Cell. 65:599-610.

17. Uitto, J., L. C. Chung-Honet, and A. M. Christiano. 1992. Molecular biology and pathology of type VII collagen. Exp. Dermatol. 1:2-10.

18. Wetzels, R. H. W., H. C. M. Robben, I. M. Leigh, H. E. Schaafsma, G. P. Vooijs, and F. C. S. Ramaekers. 1991. Distribution patterns of type VII collagen in normal and malignant human tissue. Am. J. Pathol. 139:451-459.

19. Burgeson, R. E., G. P. Lunstrum, B. Rokosova, C. S. Rimberg, L. M. 
Rosenbaum, and D. R. Keene. 1990. The structure and function of type VII collagen. Ann. NY Acad. Sci. 580:32-43.

20. Parente, M. G., L. C. Chung, J. Ryynänen, D. T. Woodley, K. C. Wynn, E. A. Bauer, M.-G. Mattei, M.-L. Chu, and J. Uitto. 1991. Human type VII collagen: cDNA cloning and chromosomal mapping of the gene. Proc. Natl. Acad. Sci. USA. 88:6931-6935.

21. Christiano, A. M., M. G. Parente, T. C. Pan, R. Z. Zhang, D. T. Woodley, L. M. Rosenbaum, M.-L. Chu, R. E. Burgeson, and J. Uitto. 1992. Cloning and characterization of human type VII collagen cDNAs. J. Invest. Dermatol. 98:552. (Abstr.).

22. Bächinger, H. P., N. P. Morris, G. P. Lunstrum, D. R. Keene, L. M. Rosenbaum, L. A. Compton, and R. E. Burgeson. 1990. The relationship of the biophysical and biochemical characteristics of type VII collagen to the function of anchoring fibrils. J. Biol. Chem. 265:10095-10101.

23. Ryynänen, M., R. G. Knowlton, M. G. Parente, L. C. Chung, M.-L. Chu, and J. Uitto. 1991. Human type VII collagen: genetic linkage of the gene (COL7A1) on chromosome 3 to dominant dystrophic epidermolysis bullosa. Am. J. Human Genet. 49:797-803.

24. Ryynänen, M., R. G. Knowlton, and J. Uitto. 1991. Mapping of epidermolysis bullosa simplex mutation to chromosome 12. Am. J. Hum. Genet. 49:978-984.

25. Bonifas, J. M., A.-L. Rothman, and E. Epstein. 1991. Epidermolysis bullosa simplex: evidence in two families for keratin gene abnormalities. Science (Wash. DC). 254:1202-1205.

26. Coulombe, P. A., M. E. Hutton, A. Letai, A. Hebert, A. S. Paller, and E. Fuchs. 1991. Point mutations in human keratin 14 genes of epidermolysis bullosa simplex patients: genetic and functional analyses. Cell. 66:1301-1311.

27. Hatzfeld, M., and K. Weber. 1990. The coiled coil of in vitro assembled keratin filaments is a heterodimer of type I and II keratins: use of site-specific mutagenesis and recombinant protein expression. J. Cell Biol. 110:1199-1210.

28. Vassar, R., P. A. Coulombe, L. Degenstein, K. Albers, and E. Fuchs. 1991. Mutant keratin expression in transgenic mice causes marked abnormalities resembling a human genetic skin disease. Cell. 64:365-380.

29. Ryynänen, M., J. Ryynänen, S. Sollberg, R. V. Iozzo, R. G. Knowlton, and J. Uitto. 1992. Genetic linkage of type VII collagen (COL7A1) to dominant dystrophic epidermolysis bullosa in families with abnormal anchoring fibrils. $J$. Clin. Invest. 89:974-980.

30. Bruckner-Tuderman, L. 1991. Collagens of the dermo-epidermal junction: role in bullous disorders. Eur. J. Dermatol. 1:89-100.

31. Chung-Honet, L. C., A. M. Christiano, A. Hovnanian, and J. Uitto. 1992.
Partial characterization of the gene for human type VII collagen (COL7A1). $J$. Invest. Dermatol. 98:567. (Abstr.).

32. Uitto, J., M. Ryynänen, A. M. Christiano, A. Hovnanian, R. Frants, E. A. Bauer, and R. G. Knowlton. 1992. Genetic linkage of the type VII collagen gene (COL7A1) to dominant dystrophic epidermolysis bullosa (DDEB) in families with abnormal anchoring fibrils. J. Invest. Dermatol. 98:576. (Abstr.).

33. Hovnanian, A., P. Duquesnoy, C. Blanchet-Bardon, R. G. Knowlton, S. Anselem, M. Lathrop, L. Dubertret, J. Uitto, and M. Goossens. 1992. Genetic linkage of recessive epidermolysis bullosa to the type VII collagen gene. J. Clin. Invest. 90:1033-1037.

34. Bauer, E. A. 1984. Collagenase in recessive dystrophic epidermolysis bullosa. Ann. NY Acad. Sci. 460:311-320.

35. Hovnanian, A., P. Duquesnoy, S. Amselem, C. Blanchet-Bardon, M Lathrop, L. Dubertret, and M. Goossens. 1991. Exclusion of linkage between the collagenase gene and generalized recessive dystrophic epidermolysis bullosa phenotype. J. Clin. Invest. 88:1716-1721.

36. Prockop, D. J. 1992. Mutations in collagen genes as a cause of connectivetissue diseases. N. Engl. J. Med. 326:540-546.

37. Leigh, I. M., I. McKay, N. Carver, H. Navsaria, and C. Green. 1991. Skin equivalents and cultured skin: from the Petri dish to the patient. Wounds. 3:141148.

38. Carter, D. M., A. N. Lin, M. C. Varghese, D. Caldwell, L. A. Pratt, and M. Eisinger. 1987. Treatment of junctional epidermolysis bullosa with epidermal autografts. J. Am. Acad. Dermatol. 17:246-250.

39. Jensen, P. K. A., and L. Bolund. 1991. Tissue culture of human epidermal keratinocytes: a differentiating model system for gene testing and somatic gene therapy. J. Cell Sci. 100:255-259.

40. Ryynänen, J., S. Sollberg, D. R. Olsen, and J. Uitto. 1991. Transforming growth factor- $\beta$ up-regulates type VII collagen gene expression in normal and transformed epidermal keratinocytes in culture. Biochem. Biophys. Res. Commun. 180:673-680.

41. Tang, D.-C., M. DeVit, and S. A. Johnson. 1992. Genetic immunization is a simple method for eliciting an immune response. Nature (Lond.). 356:152-154.

42. Compton, J. G., J. J. DiGiovanna, S. K. Santucci, K. S. Kearns, C. I. Amos, D. L. Abangan, B. P. Korge, O. W. McBride, P. M. Steinert, and S. J. Bale. 1992. Linkage of epidermolytic hyperkeratosis to the type II keratin gene cluster on chromosome 12q. Nature Genet. 1:301-305.

43. Arnemann, J., N. K. Spurr, and R. S. Buxton. 1992. The human gene (DSG3) coding for the pemphigus vulgaris antigen is, like the genes coding for the other two known desmogleins, assigned to chromosome 18. Hum. Genet. 89:347350 . 